\title{
Social and regional differences in food and alcohol consumption and their measurement in a national birth cohort
}

\author{
F E M BRADDON, ${ }^{1}$ M E J WADSWORTH, ${ }^{2} \mathrm{~J}$ M C DAVIES, ${ }^{2}$ AND H A CRIPPS ${ }^{1}$ \\ From the Department of Epidemiology and Community Medicine, ${ }^{1}$ University of Bristol, and MRC National \\ Survey of Health and Development, Department of Community Medicine, University College London and The \\ Middlesex Hospital Medical School, London ${ }^{2}$
}

SUMMARY The problem of collecting detailed dietary information on a large population scattered throughout England, Wales and Scotland was resolved by use of a 7 day dietary diary, introduced at home interviews. Information on food types and quantities was coded to provide data on a wide range of nutrients. Reported levels of iron and fibre intake were found to be particularly low in relation to current recommended daily intakes, which were more often achieved by men than by women. Best dietary habits were associated with good educational attainment, whatever the social class of origin, but in women this was in some circumstances associated also with relatively high intakes of alcohol, protein and fats. Worst dietary habits were associated with low social class of family of origin and low educational attainment. Mean intakes of some nutrients varied significantly by region, in most cases independently of class and education. The implications of these findings are discussed.

In a 36 year follow up of a national birth cohort, information on diet was required to help understand the relationship of blood pressure with body mass measured at age 36 years. It was also studied in its own right in relation to social position, education and family circumstances. This paper describes the method of dietary assessment, and gives an account of the findings and their variation in this nationally representative study population.

\section{Methods}

This birth cohort study was begun in 1946. After investigating medical and social circumstances of all births that occurred in the week 3-9th March 1946, ${ }^{1-3}$ a sample was taken for follow up. This comprised all the single, legitimate births to wives of non-manual and agricultural workers, and one in four of all the single, legitimate births to wives of manual workers. Information has been collected at intervals of 2 years or less during childhood and adolescence, and at intervals of approximately 5 years in adult life. The last contact was in 1982 at age 36 years, when 56 research nurses successfully interviewed and measured 3322 study members in their homes, $85.7 \%$ of the sample still resident in England, Wales or Scotland.
At each contact a wide range of age appropriate data was collected on medical, social, psychological, educational and occupational topics. An outline description of all the contacts ${ }^{4}$ and a list of all the publications are available. $^{5}$

A prospective 5 day diary method, with interviewer assisted recall of the 2 days immediately before interview, was used to collect dietary information. This technique is relatively inexpensive, presents fewer difficulties when food and drink are consumed away from home, and allows tolerable approximations of weights and quantities by asking for information to be given in household measures. ${ }^{67}$ It may be subject to the risk of under or over reporting because of difficulties in estimating quantities, because of a desire to give "acceptable" answers and because of individual variation in keeping such notes. However, this method, used over a period of 7 days, estimates intake with sufficient accuracy to classify individuals into groups for analysis of data on most macronutrients and a number of micronutrients. ${ }^{89}$

The diary was introduced to respondents by the research nurse, who provided instruction on its use by asking about intake of all foods during the previous 2 days, and using this information to show respondents how to complete the details. Respondents then filled in 
the diary on the next 5 days and returned it by post. Of the 3322 diaries issued at interviews, $2424(73 \%)$ were completed for 4 days or more and returned. Of these, 1284 (39\% of those issued) had been completed for the whole time period requested. These 7 day diaries were used to calculate mean intakes of each nutrient by men and women for each day of the week, and using analysis of variance weights were established for use in the estimation of intakes on the days which were not accounted for in incomplete diaries. Analysis of variance showed there were no significant differences in reports of mean intakes when the retrospective days were compared with the prospective days. There were no statistically significant differences in sex, social class or education of those who completed and returned a diary compared with those who did not.

Data on the nutrient composition of foods were obtained from standard tables, ${ }^{10}{ }^{11}$ unpublished work from the MRC's Dunn Nutrition Unit, Cambridge (personal communication), and where necessary also from manufacturers, and by using individual recipes. Portions were allocated using standard weights of household measures, and average portions obtained from a weighted intake survey in a similar age group. ${ }^{12}$ Only $6.5 \%$ of diaries had no information on portion sizes and/or insufficient description of food, and wherever possible in these instances average portion sizes were used, estimated from a weighed intake study. ${ }^{12}$ Take-away food and meals eaten in restaurants were coded from a number of weighings carried out in local area studies, using data from Nelson ${ }^{12}$ and the Dunn Nutrition Unit (personal communication). No follow up of respondents was carried out to check on diary information.

Nutrient intake for each meal and day, and the total for completed days and average daily intakes were calculated. The data presented in this paper comprise mean daily intakes of nutrients which could be reliably estimated using this method. Nutrient data were calculated from all diet diaries received but only those with four or more completed days were used in analyses $(n=2424)$ reported in this paper, with the exception of alcohol; in that case only diaries with seven completed days were used $(n=2017)$.

Table 1 Intake of selected nutrients by 36 year old men $(n=1190)$ and women $(n=1234)$, and comparisons with 4 other studies

\begin{tabular}{|c|c|c|c|c|c|c|c|c|c|}
\hline \multirow{2}{*}{$\begin{array}{l}\text { Nutrient } \\
\text { type and } \\
\text { units of } \\
\text { measurement }\end{array}$} & \multirow[b]{2}{*}{ Sex } & \multicolumn{2}{|c|}{$\begin{array}{l}\text { Observed daily } \\
\text { intake }\end{array}$} & \multicolumn{2}{|c|}{$\begin{array}{l}\text { \% Contribution of major } \\
\text { nutrients to energy intake }\end{array}$} & \multirow{2}{*}{$\begin{array}{l}\text { NFS } 13 \\
\text { data on \% } \\
\text { non-alcohol } \\
\text { energy in } \\
\text { households } \\
\text { with } \\
\text { housewife } \\
\text { aged } \\
35-44 \text { yrs }\end{array}$} & \multicolumn{2}{|c|}{$\begin{array}{l}\text { Data from } 3 \text { weighed intake } \\
\text { Scottish men }\end{array}$} & \multirow{2}{*}{$\begin{array}{l}\text { studies }^{14} \\
\text { Welsh men } \\
1979 \\
45-59 \text { yrs } \\
(n=493)\end{array}$} \\
\hline & & Mean & $S D$ & Total energy & $\begin{array}{l}\text { Non-alcohol } \\
\text { energy }\end{array}$ & & $\begin{array}{l}1976 \\
40 \text { yrs } \\
(n=97)\end{array}$ & $\begin{array}{l}1980-2 \\
45-54 \text { yrs } \\
(n=164)\end{array}$ & \\
\hline Energy (MJ) & $\stackrel{\mathbf{M}}{\mathbf{F}}$ & $\begin{array}{r}10.0 \\
7.0\end{array}$ & $\begin{array}{l}2.5 \\
1.9\end{array}$ & & & & $-^{12 \cdot 1}$ & $-^{11 \cdot 2}$ & $-^{10 \cdot 1}$ \\
\hline \multirow[t]{2}{*}{ Protein (g) } & $\mathbf{M}$ & 82.6 & $18 \cdot 7$ & $14.0(14.0)^{*}$ & $15 \cdot 1(15 \cdot 1)^{*}$ & \multirow{2}{*}{$13 \cdot 1 \dagger$} & 97 & 91 & $82 \cdot 7$ \\
\hline & $\mathbf{F}$ & $62 \cdot 3$ & $14 \cdot 4$ & $15.4(15.5)$ & $15.9(15.9)$ & & - & - & - \\
\hline \multirow[t]{2}{*}{ Fat (g) } & $\mathbf{M}$ & 103 & 28 & $38.4(38 \cdot 2)$ & $40 \cdot 4(40 \cdot 3)$ & \multirow{2}{*}{$42 \cdot 8 \dagger$} & 121 & 114 & 99.9 \\
\hline & $\mathbf{F}$ & 75 & 23 & $41 \cdot 4(41 \cdot 0)$ & $41 \cdot 6(41 \cdot 5)$ & & - & - & - \\
\hline \multirow[t]{2}{*}{ Carbohydrate (g) } & $\mathbf{M}$ & 262 & 76 & $40.8(40.9)$ & $41 \cdot 4(41 \cdot 5)$ & \multirow{2}{*}{$45 \cdot 4 \dagger$} & 305 & 298 & $277 \cdot 4$ \\
\hline & $\mathbf{F}$ & 186 & 62 & $43.7(43.9)$ & $42.5(42.6)$ & & - & - & - \\
\hline \multirow[t]{2}{*}{ Alcohol (g) } & \multirow{2}{*}{\multicolumn{2}{|c|}{$\begin{array}{l}M \\
(n=983) \\
F \\
(n=1034)\end{array}$}} & $26 \cdot 7$ & $6.6 \quad(6.9)$ & & & 40 & 26 & $19 \cdot 4$ \\
\hline & & & $10 \cdot 1$ & $2.8 \quad(2.7)$ & & & - & - & - \\
\hline Fibre (g) & $\stackrel{\mathbf{M}}{\mathbf{F}}$ & $\begin{array}{l}18.9 \\
15.1\end{array}$ & $\begin{array}{l}6.6 \\
5.8\end{array}$ & & & & $-^{17 \cdot 5}$ & $19 \cdot 8$ & $1^{19 \cdot 1}$ \\
\hline $\begin{array}{l}\text { Cereal fibre (g) } \\
\text { (included in fibre) }\end{array}$ & $\stackrel{\mathbf{M}}{\mathbf{F}}$ & $\begin{array}{l}8.7 \\
7.0\end{array}$ & $\begin{array}{l}4.7 \\
4.0\end{array}$ & & & & - & $9 \cdot 3$ & $8 \cdot 6$ \\
\hline Total sugars (g) & $\stackrel{\mathbf{M}}{\mathbf{F}}$ & $\begin{array}{l}95 \cdot 8 \\
67.2\end{array}$ & $\begin{array}{l}41 \cdot 2 \\
31 \cdot 5\end{array}$ & & & & & & \\
\hline $\begin{array}{l}\text { Added sugar (g) } \\
\text { (included in total } \\
\text { sugars) }\end{array}$ & $\stackrel{\mathbf{M}}{\mathbf{F}}$ & $\begin{array}{l}77 \cdot 7 \\
50 \cdot 3\end{array}$ & $\begin{array}{l}38 \cdot 4 \\
28 \cdot 8\end{array}$ & & & & & & \\
\hline Calcium (mg) & $\stackrel{\mathbf{M}}{\mathbf{F}}$ & $\begin{array}{l}927 \\
740\end{array}$ & $\begin{array}{l}299 \\
256\end{array}$ & & & & & & \\
\hline Iron (mg) & $\stackrel{\mathbf{M}}{\mathbf{F}}$ & $\begin{array}{l}12 \cdot 8 \\
10 \cdot 1\end{array}$ & $\begin{array}{l}3 \cdot 7 \\
3.8\end{array}$ & & & & & & \\
\hline Vitamin C (mg) & $\underset{\mathbf{F}}{\mathbf{M}}$ & $\begin{array}{l}60 \cdot 5 \\
57 \cdot 4\end{array}$ & $\begin{array}{l}33.9 \\
41.7\end{array}$ & & & & & & \\
\hline
\end{tabular}

* Figures in parentheses weighted to take account of social class stratified sampling.

$t$ Data for men and women combined 
Results

We report findings on the population distribution of intakes of a number of nutrients, from the range of all those collected on the basis of current topicality.

\section{SEX DIFFERENCES}

Table 1 shows, for men and women, the means and standard deviations of daily intake of energy and the nutrients studied, the percentage contribution of major nutrients to energy intake, and comparisons with four other studies.

Added sugar was calculated from total sugars as published in the food tables. ${ }^{10}$ Naturally occurring sugar in fruit, vegetables and milk was subtracted from total sugars to give the contents of added sugar, mainly sucrose. The added sugar content of the diet is therefore an estimate of consumption of fibre depleted refined sugar.

With the exception of calcium, mean intakes of all nutrients were significantly higher in men than in women. Twice as many women as men reported taking no alcohol during the study period. Only 30 women and three men said that they were currently on weight reducing diets, two of them on medical advice. The one man and three women who took $500 \mathrm{mg}$ or more of vitamin $\mathrm{C}$ are included in this table.
Although in comparison with National Food Survey findings ${ }^{13}$ this study population had an apparently higher protein and lower fat and carbohydrate contribution to energy intake, this was not accounted for by the sampling methods, since statistical weighting to allow for the effect of initial population sampling made very little difference to the findings, as table 1 shows. It might, however, be accounted for by differences in methods of data collection, since the study reported here is of all food consumption by individuals as compared with the household purchasing estimation methods used by the National Food Survey.

Comparisons with three weighed intake studies, in table 1, show considerable similarities, particularly with findings from the larger study.

Because of the skewed distributions of intakes of some nutrients, statistical analyses have been carried out on transformed values as appropriate, but findings presented in the tables are observed values. For men, square root transformations were used for carbohydrate, sucrose, total sugar, fibre and cereal fibre, and $\log$ to the base 10 values for vitamin $C$ and alcohol. For women, square root values were used for total sugar and cereal fibre, and log to the base 10 values for vitamin $\mathrm{C}$, sucrose, iron and alcohol.

Table 2 Population distribution of intake of selected nutrients in relation to recommended daily intakes $(R D I)$. Only nutrients for which an RDI exists are included.

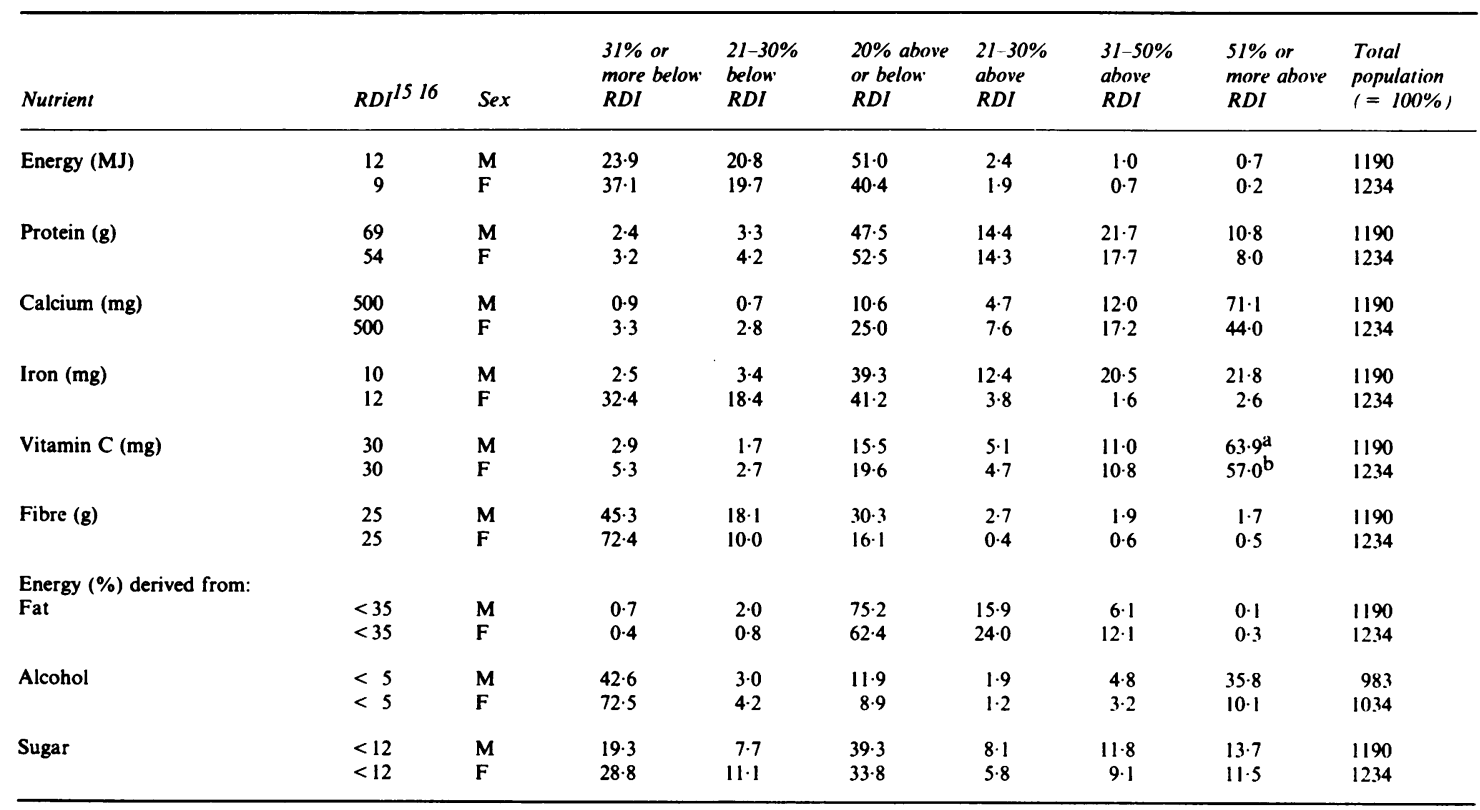

a Includes one man with mean vitamin $C$ intake $\geqslant 500 \mathrm{mg}$.

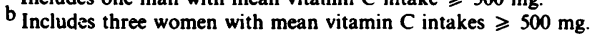


ACHIEVEMENT OF RECOMMENDED INTAKES

Recommendations have been made for daily intake (RDI) of various nutrients, ${ }^{1516}$ and the extent to which these recommended levels were achieved is shown in table 2 . Although high percentages of both men and women were taking close to the recommended or more than the recommended level of many nutrients listed in table 2, nevertheless sex differences among those whose mean intake was $20 \%$ or more below the recommended level are striking. Very much greater proportions of women than men were taking $20 \%$ or more below the recommended average daily level of iron and calcium, and almost twice as many women as men were below the RDI of vitamin C. Proportionately more men (36.6\%) than women $(17.6 \%)$ achieved or exceeded the Health Education Council's (HEC) recommended short term target for fibre intake ( $25 \mathrm{~g}$ per day). ${ }^{15}$ The lower energy and carbohydrate intakes in women made it less likely that they could achieve this recommendation. The long term target for fibre intake recommended by the $\mathrm{HEC}$ is $30 \mathrm{~g}$ per day. ${ }^{15}$
SOCIAL CLASS AND EDUCATION DIFFERENCES Using job descriptions, those gainfully employed were allocated to a Registrar General's social class. ${ }^{17}$ Table 3 shows the non-manual class tendency towards a greater intake of fibre, calcium, iron and vitamin $\mathbf{C}$ compared with those in manual occupations. Amongst men there was a relatively high intake of sugar, fat and carbohydrate in the manual social classes, and in women there was a high average intake of alcohol in those in non-manual occupations, as was found in another national study. ${ }^{18}$

Social class differences in eating habits may reflect level of education, and mean intakes of the nutrients studied are shown in tables 4 and 5 in relation to achieved educational level; all members of the population are included rather than only those who are gainfully employed. Women who attained the highest educational qualifications had significantly higher energy intakes than other women, and it is likely that this difference was the result of their significantly greater mean intakes of fats, total sugar and, in particular, alcohol. To avoid possible

Table 3 Mean daily intake of nutrients by sex and social class.

\begin{tabular}{|c|c|c|c|c|c|c|c|}
\hline & & \multicolumn{5}{|c|}{ Social class of those gainfully employed } & \multirow[b]{2}{*}{$\begin{array}{l}\text { Not gainfully } \\
\text { employed } \\
(M=72, F=489)\end{array}$} \\
\hline & & $\begin{array}{l}I \& I I \\
(M=529, F=227)\end{array}$ & $\begin{array}{l}\text { III NM } \\
(M=126, F=291)\end{array}$ & $\begin{array}{l}I I I M \\
(M=336, F=48)\end{array}$ & $\begin{array}{l}I V \& V \\
(M=111, F=172)\end{array}$ & $\begin{array}{l}\text { Significance } \\
\text { of analysis } \\
\text { of variance }\end{array}$ & \\
\hline Energy (MJ) & $\begin{array}{l}\mathbf{M} \\
\mathbf{F}\end{array}$ & $\begin{array}{l}9 \cdot 9 \\
7 \cdot 2\end{array}$ & $\begin{array}{l}9.9 \\
6.9\end{array}$ & $\begin{array}{r}10 \cdot 6 \\
7 \cdot 2\end{array}$ & $\begin{array}{r}10.4 \\
6.8\end{array}$ & $\begin{array}{l}\mathrm{p}<0.001 \\
\text { ns }\end{array}$ & $\begin{array}{l}9 \cdot 0 \\
7 \cdot 0\end{array}$ \\
\hline Protein (g) & $\begin{array}{l}\mathbf{M} \\
\mathbf{F}\end{array}$ & $\begin{array}{l}81 \cdot 8 \\
64 \cdot 6\end{array}$ & $\begin{array}{l}81 \cdot 2 \\
62 \cdot 3\end{array}$ & $\begin{array}{l}85 \cdot 2 \\
63 \cdot 5\end{array}$ & $\begin{array}{l}85 \cdot 4 \\
59 \cdot 4\end{array}$ & $\begin{array}{l}p<0.05 \\
p<0.05\end{array}$ & $\begin{array}{l}75.9 \\
61.9\end{array}$ \\
\hline Fat (g) & $\begin{array}{l}\mathbf{M} \\
\mathbf{F}\end{array}$ & $\begin{array}{r}101 \\
77\end{array}$ & $\begin{array}{r}101 \\
74\end{array}$ & $\begin{array}{r}107 \\
79\end{array}$ & $\begin{array}{r}105 \\
72\end{array}$ & $\begin{array}{l}\mathrm{p}<0.05 \\
\text { ns }\end{array}$ & $\begin{array}{l}89 \\
76\end{array}$ \\
\hline Carbohydrate (g) & $\begin{array}{l}\mathbf{M} \\
\mathbf{F}\end{array}$ & $\begin{array}{l}255 \\
184\end{array}$ & $\begin{array}{l}261 \\
188\end{array}$ & $\begin{array}{l}276 \\
183\end{array}$ & $\begin{array}{l}273 \\
183\end{array}$ & $\begin{array}{l}\mathrm{p}<0.001 \\
\text { ns }\end{array}$ & $\begin{array}{l}233 \\
191\end{array}$ \\
\hline Alcohol (g) & $\begin{array}{l}\mathbf{M} \\
\mathbf{F}\end{array}$ & $\begin{array}{r}22 \cdot 6 \\
9 \cdot 5\end{array}$ & $\begin{array}{r}21 \cdot 3 \\
7 \cdot 4\end{array}$ & $\begin{array}{r}25 \cdot 4 \\
9 \cdot 3\end{array}$ & $\begin{array}{r}22 \cdot 1 \\
5 \cdot 5\end{array}$ & $\begin{array}{l}\text { ns } \\
p<0.001\end{array}$ & $\begin{array}{r}24 \cdot 1 \\
4 \cdot 8\end{array}$ \\
\hline Fibre (g) & $\begin{array}{l}\mathbf{M} \\
\mathbf{F}\end{array}$ & $\begin{array}{l}19 \cdot 3 \\
15 \cdot 9\end{array}$ & $\begin{array}{l}19 \cdot 9 \\
15 \cdot 0\end{array}$ & $\begin{array}{l}18 \cdot 8 \\
13 \cdot 4\end{array}$ & $\begin{array}{l}18 \cdot 4 \\
14 \cdot 1\end{array}$ & $\begin{array}{l}\text { ns } \\
\mathrm{p}<0.01\end{array}$ & $\begin{array}{l}17 \cdot 1 \\
15 \cdot 4\end{array}$ \\
\hline $\begin{array}{l}\text { Cereal fibre (g) } \\
\text { (included in fibre) }\end{array}$ & $\begin{array}{l}\mathbf{M} \\
\mathbf{F}\end{array}$ & $\begin{array}{l}9 \cdot 3 \\
7 \cdot 3\end{array}$ & $\begin{array}{l}8 \cdot 9 \\
7 \cdot 1\end{array}$ & $\begin{array}{l}7 \cdot 9 \\
6 \cdot 1\end{array}$ & $\begin{array}{l}8 \cdot .5 \\
6 \cdot 1\end{array}$ & $\begin{array}{l}\mathrm{p}<0.001 \\
\mathrm{p}<0.05\end{array}$ & $\begin{array}{l}7 \cdot 5 \\
7 \cdot 2\end{array}$ \\
\hline Total sugars (g) & $\begin{array}{l}\mathbf{M} \\
\mathbf{F}\end{array}$ & $\begin{array}{l}96 \cdot 4 \\
69 \cdot 3\end{array}$ & $\begin{array}{l}94 \cdot 4 \\
64 \cdot 5\end{array}$ & $\begin{array}{r}100 \cdot 3 \\
69 \cdot 7\end{array}$ & $\begin{array}{l}91 \cdot 5 \\
64 \cdot 7\end{array}$ & $\begin{array}{l}\mathrm{p}<0.001 \\
\text { ns }\end{array}$ & $\begin{array}{l}83 \cdot 1 \\
68 \cdot 5\end{array}$ \\
\hline $\begin{array}{l}\text { Added sugar (g) } \\
\text { (included in total sugars) }\end{array}$ & $\begin{array}{l}\mathbf{M} \\
\mathbf{F}\end{array}$ & $\begin{array}{l}75 \cdot 6 \\
49 \cdot 0\end{array}$ & $\begin{array}{l}75 \cdot 9 \\
48 \cdot 0\end{array}$ & $\begin{array}{l}84 \cdot 4 \\
54 \cdot 2\end{array}$ & $\begin{array}{l}76 \cdot 4 \\
49 \cdot 6\end{array}$ & $\begin{array}{l}\mathrm{p}<0.01 \\
\text { ns }\end{array}$ & $\begin{array}{l}68 \cdot 8 \\
52 \cdot 2\end{array}$ \\
\hline Calcium (mg) & $\begin{array}{l}\mathbf{M} \\
\mathbf{F}\end{array}$ & $\begin{array}{l}946 \\
779\end{array}$ & $\begin{array}{l}929 \\
722\end{array}$ & $\begin{array}{l}935 \\
729\end{array}$ & $\begin{array}{l}907 \\
692\end{array}$ & $\begin{array}{l}\text { ns } \\
\mathrm{p}<0.01\end{array}$ & $\begin{array}{l}800 \\
752\end{array}$ \\
\hline Iron (mg) & $\begin{array}{l}\mathbf{M} \\
\mathbf{F}\end{array}$ & $\begin{array}{l}13 \cdot 2 \\
10 \cdot 7\end{array}$ & $\begin{array}{l}12 \cdot 7 \\
10 \cdot 3\end{array}$ & $\begin{array}{r}12 \cdot 7 \\
9 \cdot 9\end{array}$ & $\begin{array}{r}12 \cdot 5 \\
9 \cdot 3\end{array}$ & $\begin{array}{l}\text { ns } \\
p<0.01\end{array}$ & $\begin{array}{r}11 \cdot 1 \\
9.9\end{array}$ \\
\hline Vitamin C (mg) & $\begin{array}{l}\mathbf{M} \\
\mathbf{F}\end{array}$ & $\begin{array}{l}67 \cdot 4^{*} \\
74 \cdot 4^{*}\end{array}$ & $\begin{array}{l}60 \cdot 8 \\
60 \cdot 4 \dagger\end{array}$ & $\begin{array}{l}54.5 \\
53.6\end{array}$ & $\begin{array}{l}52 \cdot 0 \\
51 \cdot 1\end{array}$ & $\begin{array}{l}\mathrm{p}<0.001 \\
\mathrm{p}<0.001\end{array}$ & $\begin{array}{l}53 \cdot 1 \\
53 \cdot 6\end{array}$ \\
\hline
\end{tabular}

* Values after exclusion of those taking $500 \mathrm{mg}$ or more of vitamin $\mathrm{C}$ were 66.3 in men and 67.5 in women; the statistical significance is not altered.

+ Value after excluding those taking $500 \mathrm{mg}$ or more of vitamin $\mathrm{C}$ was $55 \cdot 7$; the statistical significance is not altered. 
Table 4 Means (SD) of daily averages of nutrient intake and energy sources and their association with educational qualifications.

\begin{tabular}{|c|c|c|c|c|c|c|}
\hline & & \multicolumn{4}{|c|}{ Highest educational qualification attained } & \multirow[b]{2}{*}{$\begin{array}{l}\text { Significance of } \\
\text { analysis of } \\
\text { variance for } \\
\text { each row. }\end{array}$} \\
\hline & & $\begin{array}{l}\text { None } \\
(M=411, F=456)\end{array}$ & $\begin{array}{l}\text { Minimum school } \\
\text { leaving age exam: } \\
C S E \mid " O \text { " level } \\
(M=229, F=372)\end{array}$ & $\begin{array}{l}\text { Later school } \\
\text { leaving age exam: } \\
\text { "A" level } \\
(M=320, F=285)\end{array}$ & $\begin{array}{l}\text { University } \\
\text { degrees and } \\
\text { equivalents } \\
(M=178, F=64)\end{array}$ & \\
\hline Energy (MJ) & $\begin{array}{l}\mathbf{M} \\
\mathbf{F}\end{array}$ & $\begin{array}{c}10 \cdot 2 \\
(2 \cdot 7) \\
6 \cdot 7 \\
(2 \cdot 0)\end{array}$ & $\begin{array}{c}9 \cdot 8 \\
(2 \cdot 4) \\
7 \cdot 1 \\
(1 \cdot 8)\end{array}$ & $\begin{array}{c}10 \cdot 1 \\
(2 \cdot 3) \\
7 \cdot 4 \\
(1 \cdot 7)\end{array}$ & $\begin{array}{r}9 \cdot 9 \\
(2 \cdot 1) \\
7 \cdot 7 \\
(2 \cdot 0)\end{array}$ & $\begin{array}{l}\text { ns } \\
\mathrm{p}<0.001\end{array}$ \\
\hline Protein $(g)$ & $\begin{array}{l}\mathbf{M} \\
\mathbf{F}\end{array}$ & $\begin{array}{r}83 \cdot 2 \\
(20 \cdot 9) \\
59 \cdot 4 \\
(14 \cdot 7)\end{array}$ & $\begin{array}{c}80 \cdot 7 \\
(18 \cdot 4) \\
63 \cdot 0 \\
(14 \cdot 1)\end{array}$ & $\begin{array}{r}82 \cdot 0 \\
(16 \cdot 2) \\
64 \cdot 4 \\
(12 \cdot 2)\end{array}$ & $\begin{array}{c}83 \cdot 6 \\
(16 \cdot 2) \\
68 \cdot 8 \\
(17 \cdot 8)\end{array}$ & $\begin{array}{l}\text { ns } \\
\mathrm{p}<0.001\end{array}$ \\
\hline Fats (g) & $\begin{array}{l}\mathbf{M} \\
\mathbf{F}\end{array}$ & $\begin{array}{l}104 \\
(31 \cdot 3) \\
71 \\
(23 \cdot 6)\end{array}$ & $\begin{array}{c}99 \\
(28 \cdot 2) \\
75 \\
(23 \cdot 2)\end{array}$ & $\begin{array}{l}103 \\
(26 \cdot 3) \\
80 \\
(21 \cdot 9)\end{array}$ & $\begin{array}{l}103 \\
(24 \cdot 0) \\
85 \\
(20 \cdot 7)\end{array}$ & $\begin{array}{l}\text { ns } \\
\mathrm{p}<0.001\end{array}$ \\
\hline Carbohydrate (g) & $\begin{array}{l}\mathbf{M} \\
\mathbf{F}\end{array}$ & $\begin{array}{l}267 \\
(81 \cdot 7) \\
181 \\
(65 \cdot 4)\end{array}$ & $\begin{array}{l}255 \\
(70 \cdot 3) \\
187 \\
(58 \cdot 6)\end{array}$ & $\begin{array}{l}265 \\
(75 \cdot 8) \\
192 \\
(56 \cdot 4)\end{array}$ & $\begin{array}{l}256 \\
(69 \cdot 4) \\
191 \\
(70 \cdot 6)\end{array}$ & ns \\
\hline Alcohol (g) & $\begin{array}{l}\text { M } \\
\text { F }\end{array}$ & $\begin{array}{c}22.7 \\
(28 \cdot 4) \\
(n=372) \\
4.9 \\
(8.2) \\
(n=429)\end{array}$ & $\begin{array}{c}24.8 \\
(28 \cdot 8) \\
(n=146) \\
6.7 \\
(11.0) \\
(n=263)\end{array}$ & $\begin{array}{c}23.5 \\
(25.0) \\
(n=275) \\
8.5 \\
(9.9) \\
(n=235)\end{array}$ & $\begin{array}{c}20.9 \\
(21.6) \\
(n=144) \\
9.6 \\
(13.0) \\
(n=55)\end{array}$ & $\begin{array}{l}\mathrm{ns} \\
\mathrm{p}<0.001\end{array}$ \\
\hline Fibre (g) & $\begin{array}{l}\mathbf{M} \\
\mathbf{F}\end{array}$ & $\begin{array}{l}18 \cdot 1 \\
(6 \cdot 5) \\
13 \cdot 8 \\
(5 \cdot 3)\end{array}$ & $\begin{array}{l}18 \cdot 6 \\
(6 \cdot 6) \\
15 \cdot 3 \\
(5 \cdot 9)\end{array}$ & $\begin{array}{l}19 \cdot 5 \\
(6 \cdot 5) \\
16 \cdot 3 \\
(5 \cdot 0)\end{array}$ & $\begin{array}{l}20 \cdot 7 \\
(7 \cdot 1) \\
17 \cdot 9 \\
(8 \cdot 0)\end{array}$ & $\begin{array}{l}\mathrm{p}<0.001 \\
\mathrm{p}<0.001\end{array}$ \\
\hline $\begin{array}{l}\text { Cereal fibre (g) } \\
\text { (included in fibre) }\end{array}$ & $\begin{array}{l}\text { M } \\
\text { F }\end{array}$ & $\begin{array}{c}7 \cdot 8 \\
(4 \cdot 0) \\
5 \cdot 9 \\
(3 \cdot 7)\end{array}$ & $\begin{array}{c}8 \cdot 0 \\
(4 \cdot 5) \\
7 \cdot 3 \\
(4 \cdot 1)\end{array}$ & $\begin{array}{r}9.3 \\
(4.9) \\
7 \cdot 9 \\
(3.8)\end{array}$ & $\begin{array}{c}10 \cdot 6 \\
(5 \cdot 7) \\
9 \cdot 1 \\
(4 \cdot 9)\end{array}$ & $\begin{array}{l}\mathrm{p}<0.001 \\
\mathrm{p}<0.001\end{array}$ \\
\hline Total sugars (g) & $\begin{array}{l}\mathrm{M} \\
\mathrm{F}\end{array}$ & $\begin{array}{c}94 \cdot 6 \\
(42 \cdot 6) \\
63 \cdot 3 \\
(34 \cdot 0)\end{array}$ & $\begin{array}{c}92 \cdot 9 \\
(38 \cdot 3) \\
67 \cdot 3 \\
(29 \cdot 3)\end{array}$ & $\begin{array}{c}100 \cdot 2 \\
(43 \cdot 0) \\
72 \cdot 1 \\
(28 \cdot 8)\end{array}$ & $\begin{array}{l}96 \cdot 6 \\
(34 \cdot 2) \\
71 \cdot 0 \\
(34 \cdot 1)\end{array}$ & $\begin{array}{l}\text { ns } \\
\mathrm{p}<0.001\end{array}$ \\
\hline $\begin{array}{l}\text { Sucrose (g) } \\
\text { (included in total sugars) }\end{array}$ & $\begin{array}{l}\text { M } \\
\text { F }\end{array}$ & $\begin{array}{c}79.7 \\
(39.4) \\
49.5 \\
(31.9)\end{array}$ & $\begin{array}{c}75 \cdot 5 \\
(36 \cdot 2) \\
50 \cdot 1 \\
(27 \cdot 1)\end{array}$ & $\begin{array}{c}80 \cdot 5 \\
(40 \cdot 1) \\
52 \cdot 4 \\
(26 \cdot 7)\end{array}$ & $\begin{array}{c}72.4 \\
(31.4) \\
47.9 \\
(24.6)\end{array}$ & $\begin{array}{l}\text { ns } \\
\mathrm{p}<0.01\end{array}$ \\
\hline Iron (mg) & $\begin{array}{l}\mathbf{M} \\
\mathbf{F}\end{array}$ & $\begin{array}{c}12 \cdot 4 \\
(3 \cdot 9) \\
9 \cdot 2 \\
(3 \cdot 3)\end{array}$ & $\begin{array}{l}12 \cdot 3 \\
(3 \cdot 4) \\
10 \cdot 3 \\
(3 \cdot 9)\end{array}$ & $\begin{array}{l}13.0 \\
(3.6) \\
10.8 \\
(3.7)\end{array}$ & $\begin{array}{l}13.6 \\
(3.4) \\
11.7 \\
(5.7)\end{array}$ & $\begin{array}{l}\mathrm{p}<0.001 \\
\mathrm{p}<0.001\end{array}$ \\
\hline Calcium (mg) & $\begin{array}{l}\mathbf{M} \\
\mathbf{F}\end{array}$ & $\begin{array}{l}903 \\
(306 \cdot 5) \\
674 \\
(242 \cdot 8)\end{array}$ & $\begin{array}{l}872 \\
(297 \cdot 0) \\
744 \\
(250 \cdot 9)\end{array}$ & $\begin{array}{l}964 \\
(278 \cdot 1) \\
816 \\
(250 \cdot 8)\end{array}$ & $\begin{array}{l}994 \\
(274 \cdot 4) \\
870 \\
(290 \cdot 7)\end{array}$ & $\begin{array}{l}\mathrm{p}<0.001 \\
\mathrm{p}<0.001\end{array}$ \\
\hline Vitamin C (mg) & $\begin{array}{l}\mathbf{M} \\
\mathbf{F}\end{array}$ & $\begin{array}{c}51 \cdot 5 \\
(26 \cdot 7) \\
48 \cdot 7 \\
(27 \cdot 1)\end{array}$ & $\begin{array}{c}58 \cdot 7 \\
(28 \cdot 2) \\
60 \cdot 3+ \\
(87 \cdot 4)\end{array}$ & $\begin{array}{c}65.5^{*} \\
(42.6) \\
68.6 \pm \\
(65.4)\end{array}$ & $\begin{array}{c}74 \cdot 6 \\
(34 \cdot 0) \\
69 \cdot 7 \\
(39 \cdot 3)\end{array}$ & $\begin{array}{l}p<0.001 \\
p<0.001\end{array}$ \\
\hline
\end{tabular}

* 63.8 (29.1) after exclusion of those taking $500 \mathrm{mg}$ or more of vitamin $\mathrm{C}$ : the statistical significance is not altered. $+56.0(29.6)$ after exclusion of those taking $500 \mathrm{mg}$ or more: the statistical significance is not altered.

$\$ 63.9(31.0)$ after exclusion of those taking $500 \mathrm{mg}$ or more. 
Table 5 Means (SD) of daily averages of energy intakes derived from protein, fat, carbohydrate and alcohol and their association with educational qualifications.

\begin{tabular}{|c|c|c|c|c|}
\hline $\begin{array}{l}\text { None } \\
(M=411, F=456)\end{array}$ & $\begin{array}{l}\text { Minimum school } \\
\text { leaving age exam: } \\
C S E / \text { "O" level } \\
(M=229, F=372)\end{array}$ & $\begin{array}{l}\text { Later school } \\
\text { leaving age exam: } \\
\text { "A" level } \\
(M=320, F=285)\end{array}$ & $\begin{array}{l}\text { University } \\
\text { degrees and } \\
\text { equivalents } \\
(M=178, F=64)\end{array}$ & $\begin{array}{l}\text { Significance of } \\
\text { analysis of } \\
\text { variance for } \\
\text { each row }\end{array}$ \\
\hline
\end{tabular}

\begin{tabular}{|c|c|c|c|c|c|c|}
\hline $\begin{array}{l}\text { Energy \% from: } \\
\text { Protein }\end{array}$ & $\begin{array}{l}\mathbf{M} \\
\mathbf{F}\end{array}$ & $\begin{array}{l}14 \cdot 0 \\
(2 \cdot 3) \\
15 \cdot 6 \\
(3 \cdot 7)\end{array}$ & $\begin{array}{l}14 \cdot 0 \\
(2 \cdot 2) \\
15 \cdot 5 \\
(3 \cdot 4)\end{array}$ & $\begin{array}{l}13 \cdot 8 \\
(2 \cdot 2) \\
15 \cdot 0 \\
(2 \cdot 7)\end{array}$ & $\begin{array}{l}14 \cdot 3 \\
(2 \cdot 2) \\
15 \cdot 4 \\
(2 \cdot 9)\end{array}$ & ns \\
\hline Fat & $\begin{array}{l}\mathbf{M} \\
\mathbf{F}\end{array}$ & $\begin{array}{l}38 \cdot 4 \\
(5 \cdot 2) \\
40 \cdot 3 \\
(5 \cdot 1)\end{array}$ & $\begin{array}{l}37 \cdot 9 \\
(4 \cdot 7) \\
40 \cdot 1 \\
(4 \cdot 4)\end{array}$ & $\begin{array}{l}38 \cdot 4 \\
(4 \cdot 5) \\
40 \cdot 6 \\
(4 \cdot 6)\end{array}$ & $\begin{array}{l}39 \cdot 1 \\
(4 \cdot 0) \\
42 \cdot 2 \\
(3 \cdot 9)\end{array}$ & $\begin{array}{l}\text { ns } \\
\mathrm{p}<0.01\end{array}$ \\
\hline Carbohydrate & $\begin{array}{l}\mathbf{M} \\
\mathbf{F}\end{array}$ & $\begin{array}{l}41 \cdot 3 \\
(5 \cdot 8) \\
42 \cdot 1 \\
(6 \cdot 6)\end{array}$ & $\begin{array}{l}40.9 \\
(5 \cdot 3) \\
41 \cdot 4 \\
(5 \cdot 6)\end{array}$ & $\begin{array}{l}40.9 \\
(5.8) \\
40.8 \\
(5.8)\end{array}$ & $\begin{array}{l}40 \cdot 3 \\
(5 \cdot 7) \\
38 \cdot 6 \\
(6 \cdot 0)\end{array}$ & $\begin{array}{l}\text { ns } \\
\text { p }<0.001\end{array}$ \\
\hline Alcohol & $\begin{array}{l}\mathbf{M} \\
\mathbf{F}\end{array}$ & $\begin{array}{c}6 \cdot 3 \\
(7 \cdot 2) \\
2 \cdot 1 \\
(3 \cdot 4)\end{array}$ & $\begin{array}{c}7 \cdot 2 \\
(7 \cdot 0) \\
3 \cdot 0 \\
(5 \cdot 0)\end{array}$ & $\begin{array}{r}6.9 \\
(6.8) \\
3.6 \\
(4.2)\end{array}$ & $\begin{array}{r}6.2 \\
(5.7) \\
3.7 \\
(4.9)\end{array}$ & $\begin{array}{l}\text { ns } \\
\text { p }<0.001\end{array}$ \\
\hline
\end{tabular}

distortion caused by these higher energy intakes, nutrient density per MJ was calculated. Although women with the highest qualifications had significantly higher intakes than others of fibre and cereal fibre, and of iron, calcium, and vitamin $\mathrm{C}$, calculated as a total and per $\mathrm{MJ}$, this must be set against their significantly higher alcohol and fat intakes. The picture was less clear for men. Their alcohol and sugar intakes did not vary significantly with educational attainment but, like women, the best educated men had significantly higher mean intakes, both overall and per MJ, of iron, calcium, vitamin C, fibre and cereal fibre. The higher intakes of vitamin $C$ in the best educated of both sexes reflect the greater consumption in this study population of fruit and vegetables by those with higher educational qualifications.

Higher educational attainment was associated in both men and women with a significantly higher mean intake of fibre, including cereal fibre, and of calcium and vitamin $\mathrm{C}$, whatever the social class of the family of origin. There were significantly higher mean intakes of total sugar $(p<0.001)$ and carbohydrate $(p<0.05)$ by women from manual social class families of origin who had achieved some educational attainment. Significantly higher mean intakes of iron were found in men (from non-manual families $p<0.05$, from manual families $p<0.01$ ) and women (from nonmanual families $p<0.05$, from manual families $\mathrm{p}<0.001$ ) with higher educational qualifications. Percentage sources of energy derived from protein, fat and carbohydrates did not differ significantly, but amongst women there was a higher percentage of alcohol as a source of energy in those with highest educational qualifications $(p<0.05)$.

Thus, women from manual class families with no educational qualifications were conspicuously in the poorest nutritional position in terms of intakes of calcium, iron, cereal fibre, carbohydrate, and vitamin $C$; and in this study $26.5 \%$ of all women come from this kind of social background.

\section{REGIONAL DIFFERENCES}

Tables 6 and 7 show regional differences in intakes of nutrients. Regions in this table are the Standard Regions used by the Registrar General. ${ }^{19}$ For men the mean intakes of energy, protein, fat and carbohydrate were lowest in the southeast of England, and men reported significantly lower intakes of total fibre in Scotland and of cereal fibre in Wales. Women in Scotland and the southeast of England had the highest mean fat intake, and those in southeast England also had the highest intake of fibre, calcium and vitamin $\mathrm{C}$. There was a notably lower mean intake of vitamin $C$ and fibre amongst women in the north of England. For a number of nutrients, intakes were relatively low in women who lived in Wales, notably in energy, protein, fat, fibre including cereal fibre, and calcium.

Mean intakes amongst the best educated (university entrance or equivalents, and above) differed very little interregionally, and in this group only men in the North of England had significantly higher mean intakes of sucrose $(t=2.40$ with $\infty$ d.f., $p<0.02)$ and total sugar $(\mathrm{t}=1.96$ with $\infty$ d.f., $\mathrm{p}=0.05)$ compared with men in London and the South East. There were no significant interregional differences amongst better 
Table 6 Regional differences in means (SD) of daily averages of nutrient intakes.

\begin{tabular}{|c|c|c|c|c|c|c|c|c|}
\hline & & \multicolumn{4}{|l|}{ England } & \multirow{2}{*}{$\begin{array}{l}\text { Wales } \\
\begin{array}{l}(M=66 \\
F=62)\end{array}\end{array}$} & \multirow{2}{*}{$\begin{array}{l}\text { Scotland } \\
\\
\begin{array}{l}(M=120 \\
F=118)\end{array}\end{array}$} & \multirow[b]{2}{*}{$\begin{array}{l}\text { Significance } \\
\text { of analysis } \\
\text { of variance } \\
\text { for each row }\end{array}$} \\
\hline & & $\begin{array}{l}\text { North, } \\
\text { Yorks } \\
\text { and } \\
\text { Humberside } \\
(M=290, \\
F=247)\end{array}$ & $\begin{array}{l}\text { Midlands } \\
\text { and } \\
\text { East } \\
\text { Anglia } \\
(M=240, \\
F=279)\end{array}$ & 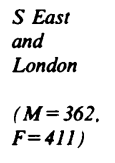 & $\begin{array}{l}S \text { West } \\
\\
(M=112 \\
F=117)\end{array}$ & & & \\
\hline Energy (MJ) & $\mathbf{M}$ & $\begin{array}{c}10.4 \\
(2.6) \\
7.0 \\
(1.9)\end{array}$ & $\begin{array}{c}10 \cdot 2 \\
(2 \cdot 4) \\
6 \cdot 8 \\
(1.9)\end{array}$ & $\begin{array}{c}9 \cdot 6 \\
(2 \cdot 2) \\
7 \cdot 1 \\
(1 \cdot 9)\end{array}$ & $\begin{array}{r}10 \cdot 4 \\
(2 \cdot 6) \\
7 \cdot 0 \\
(1 \cdot 7)\end{array}$ & $\begin{array}{c}10 \cdot 1 \\
(2 \cdot 6) \\
6 \cdot 4 \\
(2 \cdot 1)\end{array}$ & $\begin{array}{c}10 \cdot 0 \\
(2 \cdot 7) \\
7 \cdot 3 \\
(2 \cdot 0)\end{array}$ & $\begin{array}{l}\mathrm{p}<0.01 \\
\mathrm{p}<0.05\end{array}$ \\
\hline Protein (g) & $\begin{array}{l}\mathbf{M} \\
\mathbf{F}\end{array}$ & $\begin{array}{c}84.5 \\
(19.6) \\
62.6 \\
(14.6)\end{array}$ & $\begin{array}{c}83.8 \\
(19.9) \\
61.5 \\
(13.9)\end{array}$ & $\begin{array}{c}80 \cdot 0 \\
(16 \cdot 6) \\
63 \cdot 3 \\
(14 \cdot 7)\end{array}$ & $\begin{array}{c}81 \cdot 8 \\
(18 \cdot 3) \\
61.6 \\
(12 \cdot 2)\end{array}$ & $\begin{array}{c}83 \cdot 3 \\
(18.8) \\
56 \cdot 7 \\
(14.4)\end{array}$ & $\begin{array}{c}84 \cdot 3 \\
(19 \cdot 8) \\
63 \cdot 3 \\
(15 \cdot 7)\end{array}$ & $\begin{array}{l}p<0.05 \\
p<0.05\end{array}$ \\
\hline Fat (g) & $\mathbf{M}$ & $\begin{array}{c}105 \cdot 6 \\
(30 \cdot 9) \\
75 \cdot 3 \\
(23 \cdot 2)\end{array}$ & $\begin{array}{c}102.5 \\
(26 \cdot 4) \\
72.5 \\
(23.4)\end{array}$ & $\begin{array}{c}100 \cdot 0 \\
(25 \cdot 2) \\
76 \cdot 9 \\
(23 \cdot 6)\end{array}$ & $\begin{array}{c}108 \cdot 4 \\
(30 \cdot 9) \\
75 \cdot 8 \\
(20 \cdot 1)\end{array}$ & $\begin{array}{c}103.0 \\
(29 \cdot 3) \\
67 \cdot 8 \\
(25 \cdot 1)\end{array}$ & $\begin{array}{c}102 \cdot 4 \\
(31 \cdot 2) \\
78 \cdot 9 \\
(23 \cdot 5)\end{array}$ & $p<0.01$ \\
\hline Carbohydrate (g) & $\mathbf{M}$ & $\begin{array}{l}269 \\
(32 \cdot 5) \\
185 \\
(61 \cdot 6)\end{array}$ & $\begin{array}{l}272 \\
(75 \cdot 9) \\
182 \\
(59 \cdot 5)\end{array}$ & $\begin{array}{l}246 \\
(66 \cdot 2) \\
187 \\
(60 \cdot 1)\end{array}$ & $\begin{array}{l}275 \\
(78 \cdot 5) \\
184 \\
(58 \cdot 9)\end{array}$ & $\begin{array}{l}261 \\
(76 \cdot 4) \\
173 \\
(67 \cdot 4)\end{array}$ & $\begin{array}{l}261 \\
(81 \cdot 2) \\
197 \\
(71 \cdot 2)\end{array}$ & $\begin{array}{l}\mathrm{p}<0.001 \\
\text { ns }\end{array}$ \\
\hline Alcohol (g) & $\mathbf{M}$ & $\begin{array}{c}24 \cdot 4 \\
(25 \cdot 4) \\
(\mathrm{n}=212) \\
6 \cdot 5 \\
(8 \cdot 9) \\
(\mathrm{n}=210)\end{array}$ & $\begin{array}{c}22 \cdot 6 \\
(24 \cdot 7) \\
(n=201) \\
5 \cdot 6 \\
(9 \cdot 9) \\
(n=216)\end{array}$ & $\begin{array}{c}24 \cdot 0 \\
(30 \cdot 3) \\
(n=310) \\
\quad 7 \cdot 5 \\
(11 \cdot 3) \\
(n=346)\end{array}$ & $\begin{array}{c}19 \cdot 0 \\
(19 \cdot 2) \\
(n=100) \\
6 \cdot 3 \\
(10 \cdot 7) \\
(n=112)\end{array}$ & $\begin{array}{c}21.8 \\
(22.0) \\
(n=56) \\
5.9 \\
(8.0) \\
(n=50)\end{array}$ & $\begin{array}{c}22 \cdot 9 \\
(29 \cdot 7) \\
(n=104) \\
6 \cdot 3 \\
(8 \cdot 5) \\
(n=100)\end{array}$ & ns \\
\hline Total fibre (g) & $\mathbf{M}$ & $\begin{array}{l}19.0 \\
(6 \cdot 8) \\
14.0 \\
(5 \cdot 0)\end{array}$ & $\begin{array}{l}19 \cdot 5 \\
(7 \cdot 0) \\
14.9 \\
(5 \cdot 2)\end{array}$ & $\begin{array}{l}18 \cdot 5 \\
(6 \cdot 3) \\
15 \cdot 9 \\
(6 \cdot 2)\end{array}$ & $\begin{array}{l}20 \cdot 8 \\
(7 \cdot 2) \\
15 \cdot 6 \\
(6 \cdot 0)\end{array}$ & $\begin{array}{l}18 \cdot 6 \\
(5 \cdot 5) \\
13.8 \\
(5 \cdot 6)\end{array}$ & $\begin{array}{l}18 \cdot 1 \\
(6 \cdot 2) \\
15 \cdot 1 \\
(6 \cdot 0)\end{array}$ & $\begin{array}{l}\mathrm{p}<0.02 \\
\mathrm{p}<0.001\end{array}$ \\
\hline $\begin{array}{l}\text { Cereal fibre (g) } \\
\text { (included in total fibre) }\end{array}$ & $\mathbf{M}$ & $\begin{array}{r}8.9 \\
(4.7) \\
6.7 \\
(3.9)\end{array}$ & $\begin{array}{r}8.8 \\
(4.8) \\
6 \cdot 7 \\
(3 \cdot 5)\end{array}$ & $\begin{array}{r}8.4 \\
(4.4) \\
7.5 \\
(4 \cdot 4)\end{array}$ & $\begin{array}{c}9.9 \\
(6 \cdot 0) \\
7 \cdot 1 \\
(3.9)\end{array}$ & $\begin{array}{c}7.5 \\
(3.8) \\
5.8 \\
(4.0)\end{array}$ & $\begin{array}{r}8 \cdot 1 \\
(4 \cdot 2) \\
7 \cdot 2 \\
(3 \cdot 9)\end{array}$ & $\begin{array}{l}p<0.01 \\
p<0.05\end{array}$ \\
\hline Total sugars (g) & $\mathbf{M}$ & $\begin{array}{c}97 \cdot 2 \\
(44 \cdot 6) \\
66 \cdot 2 \\
(32 \cdot 2)\end{array}$ & $\begin{array}{c}99 \cdot 8 \\
(41 \cdot 1) \\
66 \cdot 4 \\
(30 \cdot 2)\end{array}$ & $\begin{array}{c}92 \cdot 6 \\
(36 \cdot 3) \\
69 \cdot 4 \\
(31 \cdot 0)\end{array}$ & $\begin{array}{c}102 \cdot 2 \\
(41 \cdot 0) \\
65 \cdot 1 \\
(28 \cdot 8)\end{array}$ & $\begin{array}{c}93 \cdot 7 \\
(39 \cdot 2) \\
58.6 \\
(31 \cdot 7)\end{array}$ & $\begin{array}{c}89 \cdot 2 \\
(46 \cdot 4) \\
69 \cdot 7 \\
(36 \cdot 4)\end{array}$ & $\begin{array}{l}\mathrm{p}<0.05 \\
\mathrm{~ns}\end{array}$ \\
\hline $\begin{array}{l}\text { Added sugar (g) } \\
\text { (included in total sugars) }\end{array}$ & $\mathbf{M}$ & $\begin{array}{c}80 \cdot 0 \\
(40 \cdot 9) \\
52 \cdot 1 \\
(30 \cdot 2)\end{array}$ & $\begin{array}{c}81 \cdot 6 \\
(38 \cdot 2) \\
49 \cdot 6 \\
(28 \cdot 2)\end{array}$ & $\begin{array}{c}73.8 \\
(34 \cdot 4) \\
51 \cdot 1 \\
(28 \cdot 3)\end{array}$ & $\begin{array}{c}83.4 \\
(39.5) \\
47.8 \\
(26.9)\end{array}$ & $\begin{array}{c}76.6 \\
(38.4) \\
44.8 \\
(29.4)\end{array}$ & $\begin{array}{l}71 \cdot 1 \\
(42 \cdot 0) \\
50 \cdot 8 \\
(30 \cdot 5)\end{array}$ & $\begin{array}{l}\mathrm{p}<0.02 \\
\mathrm{~ns}\end{array}$ \\
\hline Iron (mg) & $\mathbf{M}$ & $\begin{array}{c}12.9 \\
(4.0) \\
9.9 \\
(4.8)\end{array}$ & $\begin{array}{c}12 \cdot 6 \\
(3 \cdot 8) \\
9 \cdot 8 \\
(3 \cdot 2)\end{array}$ & $\begin{array}{l}12.8 \\
(3.6) \\
10.5 \\
(3.9)\end{array}$ & $\begin{array}{l}13 \cdot 1 \\
(3 \cdot 5) \\
10 \cdot 1 \\
(3 \cdot 2)\end{array}$ & $\begin{array}{c}12 \cdot 3 \\
(3 \cdot 0) \\
9 \cdot 2 \\
(3 \cdot 5)\end{array}$ & $\begin{array}{l}13.0 \\
(3.9) \\
10.0 \\
(3.2)\end{array}$ & $\begin{array}{l}\text { ns } \\
p<0.01\end{array}$ \\
\hline Calcium (mg) & $\mathbf{M}$ & $\begin{array}{l}941 \\
(314 \cdot 7) \\
722 \\
(230 \cdot 4)\end{array}$ & $\begin{array}{l}981 \\
(328 \cdot 6) \\
738 \\
(272 \cdot 6)\end{array}$ & $\begin{array}{l}892 \\
(259 \cdot 3) \\
781 \\
(253 \cdot 1)\end{array}$ & $\begin{array}{l}965 \\
(297 \cdot 8) \\
731 \\
(228 \cdot 8)\end{array}$ & $\begin{array}{l}848 \\
(262 \cdot 3) \\
599 \\
(255 \cdot 7)\end{array}$ & $\begin{array}{l}904 \\
(312 \cdot 1) \\
727 \\
(276 \cdot 7)\end{array}$ & $\begin{array}{l}p<0.01 \\
p<0.001\end{array}$ \\
\hline Vitamin C (mg) & $\mathbf{M}$ & $\begin{array}{c}57.0 \\
(31 \cdot 1) \\
47.6 \\
(24.7)\end{array}$ & $\begin{array}{c}61 \cdot 3 \\
(32.5) \\
56.8 \\
(31.0)\end{array}$ & $\begin{array}{c}63 \cdot 1^{*} \\
(40 \cdot 4) \\
66 \cdot 8 \dagger \\
(87 \cdot 6)\end{array}$ & $\begin{array}{r}63.4 \\
(27.4) \\
58.4 \\
(31 \cdot 6)\end{array}$ & $\begin{array}{c}59.1 \\
(29.8) \\
50.6 \\
(27.8)\end{array}$ & $\begin{array}{c}57.0 \\
(27.5) \\
63.0 \\
(85.7)\end{array}$ & $\begin{array}{l}\mathrm{p}<0.001 \\
\mathrm{p}<0.001\end{array}$ \\
\hline
\end{tabular}

* 61.6 (SD 27.8) after exclusion of those taking $500 \mathrm{mg}$ or more of vitamin C: the statistical significance is not altered.

+61.8 (SD 32.4) after exclusion of those taking $500 \mathrm{mg}$ or more of vitamin $\mathrm{C}$ : the statistical significance is not altered. 
Table 7 Regional differences in means (SD) of daily averages of energy sources

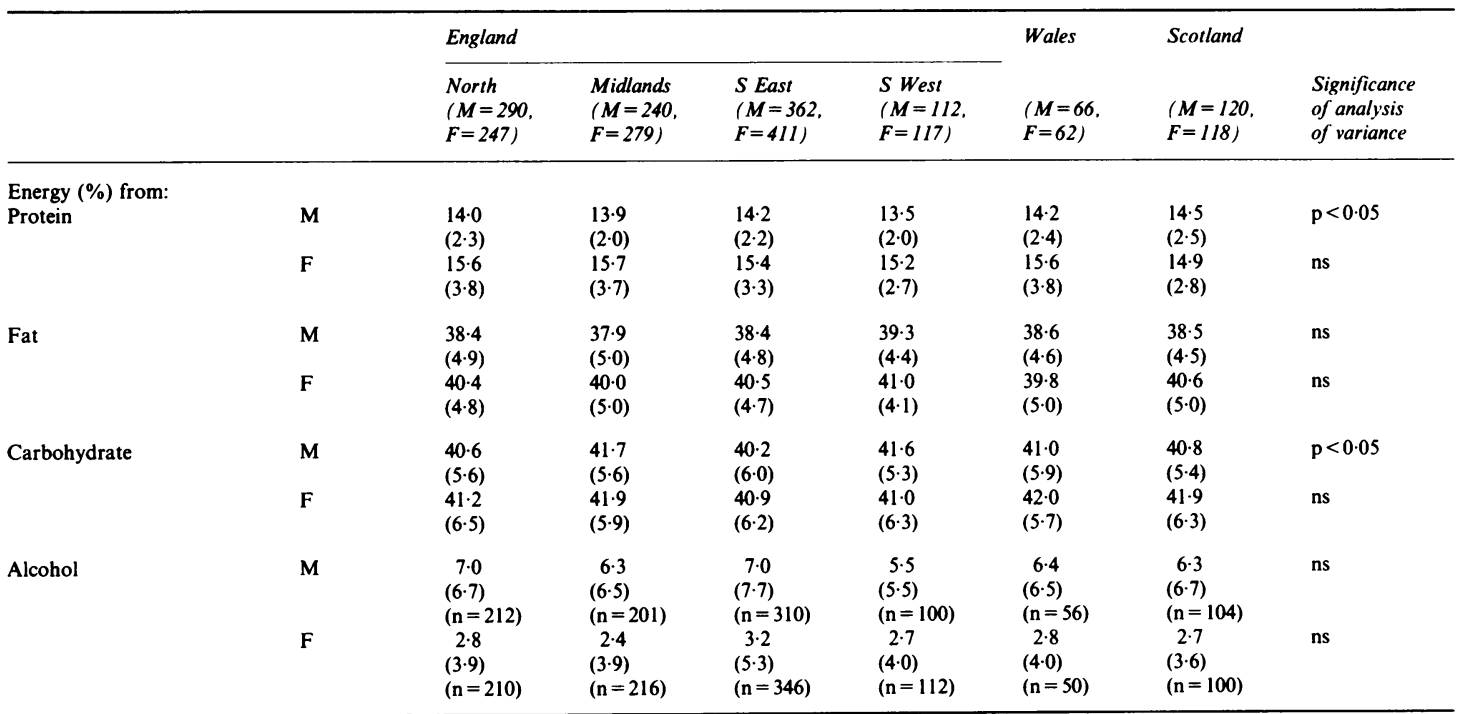

educated women. Compared with similarly educated women in London and the South East those in Wales had significantly ( $p<0.05$ or less) lower mean intakes of calcium, fibre including cereal fibre, fat, protein and energy.

Since there were regional differences in social class, particularly of the families of origin, as well as in educational attainment of men and women, stepwise multiple regression analyses were carried out to see whether these differences added to the variance explained by social class and educational attainment. A separate analysis was carried out for each sex for each nutrient, using a forced order regression which first took into account social class of family of origin and then educational attainment, before region of residence; this forced ordering was used to keep the chronological order of influences of the variables. Education was associated significantly and independently with the intakes of total fibre, cereal fibre, total sugar, iron, calcium and vitamin $\mathrm{C}$, in both sexes, at the $p<0.05$ level or less. Region of residence was independently associated, in both sexes, with carbohydrate, alcohol, sucrose, calcium and vitamin $\mathrm{C}$, and in men also with total fibre, cereal fibre and total sugar ( $<<0.05$ or less). Thus, the data on region added notably to the explained variance in most of the individual nutrients, and therefore also to the explained variance in total energy, protein and fat intakes.

\section{Discussion}

The diary method of data collection was found to be appropriate for a large national population without undue bias of sex, class or education. Findings compared well with those from three 7 day weighed intake surveys, ${ }^{14}$ mean intakes of energy, protein, fat, carbohydrate, alcohol and fibre all being closely similar. This seems therefore to be a useful form of data collection in large populations for many, but not all, nutrients. Coding is a considerable task since food items are of the order of 300 per week for each individual.

Just over half the women did not achieve the RDI of iron, and well over half the population were below the RDI for fibre. Women were more inclined than men to be deficient, by current RDI standards, in all the nutrients studied here for which there was an RDI standard.

Signs of dietary awareness, in terms of higher mean intakes of fibre, iron, calcium and vitamin $\mathrm{C}$, were associated with non-manual employment and with educational attainment; obesity was also less common in this group. ${ }^{20}$ Amongst women, however, nonmanual employment and good educational attainment were also associated with higher mean intakes of alcohol, protein and fats. These educational differences were found whatever the social class of family of origin, but women from manual class families of origin who had obtained the highest educational qualifications had intakes of alcohol more than twice as great as those from similar class backgrounds who had no qualifications. Nevertheless their intake of alcohol was still less than half the mean intake amongst men.

Regional differences were found in mean intakes of most nutrients, with the exception of alcohol, total sugar and iron, and for most nutrients social class of 
origin, level of educational attainment and region of residence each added independently to the amount of variance explained.

Evidently some influence of childhood circumstances continued, and although the experience of education had apparently improved the nutritional habits of those from lower social class families of origin, this was unlikely to have been effective in childhood, during the period of fastest growth. Only $18.9 \%$ of women from manual class families of origin attained the level of education associated, in this study, with better dietary intake. It was not surprising, therefore, to find social class differences in height, ${ }^{21} 22$ and a relative underachievement of growth potential (unpublished data) amongst men and women from manual families of origin compared with those from non-manual families. In terms of their children's lives this improved dietary awareness is clearly an advantage to the relatively small proportion of the population concerned. This study continues to investigate these and other childhood factors that are associated with health in adult life.

We would like especially to thank Professor $\mathbf{J} R \mathbf{T}$ Colley for his advice and encouragement, and Janet Wingfield and Joan Josey for their invaluable help with programming and data processing.

Address for correspondence and reprints: $\mathrm{Dr} \mathbf{M}$ Wadsworth, MRC National Survey of Health and Development, University College London and the Middlesex Hospital Medical School, Department of Community Medicine, 66-72 Gower Street, London WC1E 6EA.

\section{References}

${ }^{1}$ Joint Committee. Maternity in Great Britain. Oxford: Oxford University Press, 1948.

${ }^{2}$ Douglas JWB, Rowntree G. Supplementary maternal and child health services, part I, postnatal care, and part II, Nurseries. Pop Studies 1949; 3: 205-26.

${ }^{3}$ Douglas JWB. The health and survival of children in different social classes: the results of a national survey. Lancet 1951; ii: 440-6.

${ }^{4}$ Atkins E, Cherry N, Douglas JWB, Kiernan KE, Wadsworth MEJ. The 1946 British birth cohort: an account of its origins, progress and results. In: Mednick SA, Baert AE, eds. Prospective longitudinal research. Oxford: Oxford University Press, 1981.
${ }^{5}$ Wadsworth MEJ. Follow-up of the first British national birth cohort. Paediatr Perinatal Epidemiol 1987; 1: 95-117.

${ }^{6}$ Marr JW. Individual dietary surveys: purposes and methods. World Rev Nutr Diet 1971; 13: 105-64.

${ }^{7}$ Nettleton PA, Day KC, Nelson M. Dietary survey methods. 2. A comparison of nutrient intakes within families assessed by household measures and the semiweighed method. J Human Nutr 1980; 34: 349-54.

${ }^{8}$ Black AE. Pitfalls in dietary assessment. In: Howard A, McClean Baird I, eds. Recent advances in clinical nutrition. London: John Libbey, 1981.

${ }^{9}$ Marr JW, Heady JA. Within- and between-person variation in dietary surveys: number of days needed to classify individuals. Human Nutr Appl Nutr 1986; 40A: 347-64.

${ }^{10}$ Paul AA, Southgate DAT. McCance and Widdowson's The Composition of Foods. London: HMSO, 1978.

11 Wiles SJ, Nettleton PA, Black AE, Paul AA. The nutrient composition of some cooked dishes eaten in Britain: a supplementary food composition table. J Hum Nutr 1980; 34: 189-223.

12 Nelson M. A dietary survey method for measuring family food purchases and individual nutrient intakes concurrently, and its use in dietary surveillance. PhD Thesis. University of London.

${ }^{13}$ Ministry of Agriculture, Fisheries and Food. Household Food Consumption and Expenditure: 1982. London: HMSO, 1984.

14 Thomson M, Fulton M, Wood DA, Brown S, Elton RA, Birtwhistle A, Oliver M. A comparison of the nutrient intake of some Scotsmen with dietary recommendations. Human Nutr Appl Nutr 1985; 39A: 443-55.

15 The Health Education Council. Proposals for nutritional guidelines for health education in Britain (NACNE). London: Health Education Council, 1983.

16 Department of Health and Social Security. Recommended daily amounts of food energy and nutrients for groups of people in the United Kingdom. Reports on Health and Social Subjects, No. 15. London: HMSO, 1979.

${ }^{17}$ Classification of Occupations, 1980. London: Office of Population Censuses and Surveys, 1980.

18 The Health and Lifestyle Survey. London: Health Promotion Research Trust, 1987.

19 Office of Population Censuses and Surveys. Census 1981. National Report. Great Britain. London: HMSO, 1983.

20 Braddon FEM, Rodgers B, Wadsworth MEJ, Davies JMC. Onset of obesity in a 36 year birth cohort study. Br Med J 1986; 293: 299-303.

${ }^{21}$ Douglas JWB, Simpson HR. Height in relation to puberty, family size and social class: a longitudinal study. Milbank Memorial Fund Quarterly 1964; 42: 20-35.

22 Wadsworth MEJ. Serious illness in childhood and its association with later life achievement. In: Wilkinson RA, ed. Class and health. London: Tavistock Publications, 1986. 